\title{
Region Merging Strategy Using Statistical Analysis for Interactive Image Segmentation on Dental Panoramic Radiographs
}

\author{
Agus Zainal Arifin ${ }^{1}$, Rarasmaya Indraswari ${ }^{1}$, Nanik Suciati ${ }^{1}$, Eha Renwi Astuti ${ }^{2}$, \\ Dini Adni Navastara ${ }^{1}$
}

\begin{abstract}
In low contrast images such as dental panoramic radiographs, the optimum parameters for automatic image segmentation is not easily determined. Semi-automatic image segmentation which is interactively guided by user is one alternative that could provide a good segmentation results. In this paper we proposed a novel strategy of region merging in interactive image segmentation using discriminant analysis on dental panoramic radiographs. A new similarity measurement among regions is introduced. This measurement merges regions which have minimal inter-class variance either with object or background cluster. Since the representative sample regions are selected by user, the similarity between merged regions with the corresponded samples could be preserved. Experimental results show that the proposed region merging strategy give a high segmentation accuracy both for low contrast and natural images. Copyright $(9) 2017$ The Authors.

Published by Praise Worthy Prize S.r.l. This article is open access published under the CC BY-NC-ND license (http://creativecommons.org/licenses/by-nc-nd/3.0/).
\end{abstract}

Keywords: Dental Panoramic Radiograph, Interactive Image Segmentation, Low Contrast, Region Merging, Statistical Analysis

\section{Nomenclature}

I

$C$

$O$

$B$

$K$

$\omega_{k}$

$\mu_{k}$

$\sigma_{I}^{2}$

Dist $_{\text {OCk }}$

Dist $_{B C k}$

$t^{*}$

Input image

Non-marked cluster

Object cluster

Background cluster

Total number of regions

Mean intensity of region $k$

Inter-class variance object cluster $O$ background cluster $B$
Ratio of area between region $k$ and the image

Distance between non-marked region $C_{k}$ and

Distance between non-marked region $C_{k}$ and

Index of region with minimal distance value

\section{Introduction}

Image segmentation is an important part of medical image processing. Segmentation is usually used to separate the object of an image from its background [1]. If the object and its background cannot be separated completely then the features or information that provided by the image will be inaccurate which can be fatal in clinical application. Therefore, developing a robust and efficient algorithm for medical image segmentation is a demanding research area that continues to grow [2].

There are many image segmentation methods that have been developed. In general, image segmentation methods can be divided into three categories, namely automatic, semi-automatic, and manual image segmentation [3]. Automatic image segmentation usually use the color, texture, or shape features from the image. For some fields, such as segmentation of satellite images, amplitude and frequency modulation (AM-FM) features are extracted from the image [4]. Those features will be added by certain parameters or weight for automatic segmentation process. Usually, user has to use different optimal value of parameters to achieve satisfying segmentation results, especially in low contrast and natural images [5].

Dental panoramic radiographs is a medical images obtained from 2D X-ray examination that captures the entire mouth in a single image. Dental panoramic radiographs can be used to diagnose caries, periodontal disease, periapical pathology, osteoporosis, etc. However, dental panoramic radiograph has low contrast, therefore it is difficult to separate object and background on dental panoramic radiograph using automatic segmentation method. In addition, dental panoramic radiographs usually contaminated by speckle or multiplicative noise from X-ray imaging that makes automatic segmentation difficult. Preliminary noise reduction process using filter, such as Lee filter, Kuan filter, Weiner filter or NLM filter, is capable of reducing noise variance significantly while maintaining the mean value of the image that was contaminated by speckle noise [6]. Meanwhile, segmentation performance of low contrast and noisy images can be greatly improved if the automatic segmentation method is integrated with more 
feature information provided by user [7].

Hence, several semi-automatic image segmentation methods that combine manual and automatic segmentation by invoking the aid of user to supply information for the segmentation system have been developed. In semi-automatic image segmentation or socalled interactive image segmentation, user can interact by providing input that help the system to extract objects of the given image. Using interactive image segmentation, low contrast images can be segmented more accurately because there is another information, beside color or texture information, that provided by user to help the system to do segmentation process.

User marks several regions as samples based on consideration that the samples is the most representative regions of the background or the object in the image. A merging strategy which restricts the comparison only among adjacent regions seems to be single linkage phenomena which may result undesirable chain effect. This effect will maximize the homogeneity of either object or background cluster groups produced by the merge of a region with the representative regions. A cluster has good separability measure because it is maximized the inter-class difference and minimized the intra-class spread. In fact, maximizing intra-class variance and minimizing inter-class variance is equivalent to one another [8]. Since inter-class variance use first order statistics while intra-class variance use second order statistics, for simplicity, Otsu recommended to use inter-class variance [9]. This stand point motivated us to study a region merging strategy that can preserves maximum inter-class variance between the merged region and the representative regions chosen by the user.

In this paper we proposed a novel strategy of region merging in interactive image segmentation using discriminant analysis on dental panoramic radiographs. A new similarity measurement among regions is introduced. This measurement merges regions which have minimal inter-class variance either with object or background cluster. Since the representative sample regions are selected by user, the similarity between merged regions with the corresponded samples could be preserved. This region merging strategy is applied to low contrast images such as dental panoramic radiographs and gives satisfying results both for low contrast images and natural images.

The rest of this paper is organized as follows. In Section II, various related researches about interactive image segmentation and applications of dental panoramic radiographs will be discussed. The proposed region merging strategy will be introduced in Section III. The experimental results of the proposed method and its comparison with other methods will be reported in Section IV. Finally, conclusions are drawn in Section V.

\section{Related Research}

\section{II.1. Interactive Image Segmentation}

Algorithmic approaches to interactive image segmentation methods can be classified broadly into several classes as shown in Table I [10]. In classifiers method, graph model, and hierarchical method the image will be divided into several small regions and then those small regions will be merged to get the object and the background regions of the image. This process is called region merging. A region will be merged with another region if those two regions have similar characteristics. Distance measurement of the features between two regions is usually used to measure the similarity between those regions. The measured features can be color or texture features of the image.

TABLE I

ALGORITHMIC APPROACHES TO SEMI-AUTOMATIC IMAGE SEGMENTATION METHODS

\begin{tabular}{ll}
\hline \hline \multicolumn{1}{c}{ Method } & \multicolumn{1}{c}{ Example Algorithm } \\
\hline Region Growing & Seeded region growing ([11], [12]) \\
Deformable methods & Active contour (snakes) ([13]) \\
Graph Model & Interactive Graph Cut ([14]-[16]) \\
Hierarchical & Interactive segmentation using binary \\
& partition trees ([17], [18]) \\
Classifiers & Maximal Similarity Based Region \\
& Merging (MSRM) ([1], [19]-[21]) \\
\hline \hline
\end{tabular}

\section{II.2. Classifiers Method}

Ning et al. proposed maximal similarity based region merging (MSRM) method for region merging process in interactive image segmentation [1]. This method using histogram value of RGB color features of the image to calculate the similarity between two regions using Bhattacharyya coefficient. If the first region has the highest similarity with the second region compared to another, then the first and second region will merge. This method is need the user to mark several regions as object and background. The merging process is done by merging the adjacent non-marked region into the background region. If there are no more adjacent regions that can be added to the background regions, then the remaining non-marked regions will be added to the object regions.

Zeng et al. proposed an auto-marking image segmentation algorithm based manifold ranking (AISMR) [19]. This research use Simple Linear Interactive Clustering (SLIC) algorithm for image presegmentation. Marks for object region are obtained automatically using saliency detection. Marks for background region are obtained using combination of background priors and manifold ranking. Then the final object segmentation is done using MSRM algorithm.

Sankoh et al. proposed Extracted Pixels Similarity Features (EPSF) method for interactive image segmentation [20]. This method combined RGB and HSV color features and Gabor texture feature to get significant features in measuring distance among regions using Euclidean distance. In this paper, the region merging process was accomplished by MSRM algorithm. This method gave an accurate result, however the region merging process was done to the adjacent regions of the 
regions that marked as background. If there are any background regions which are separated completely by the object and did not marked by the user, then those background regions will be labeled as object.

Furthermore, semi-automatic segmentation has been developed for object segmentation in video. In video, an object moved to certain direction from current to its subsequent frame, create a motion vector space. Basuki et al. proposed an approach to estimate the constraints of object in video using semi-automatic [21]. First, user marked the objects in current frame as constraint coordinates. Then the motion vector is calculated using Block Matching Algorithm (BMA) and the result is added to the constraint coordinates of object in current frame to create the constraint coordinates in subsequent frame.

\section{II.3. Dental Panoramic Radiographs}

Imaging techniques in dentistry can be categorized as two-dimensional (2-D) and three-dimensional (3-D) [22]. Dental panoramic radiograph is $2-\mathrm{D}$ medical imaging technique using X-ray that is used for teeth and jaw examination in dental clinics around the world since introduced in 1950.

Dental panoramic radiographs can be used to provide information of internal structure of teeth and bone for diagnose caries, periodontal disease, periapical pathology, etc. It also has great potential as a screening tool for osteoporosis due to its widespread use, practicality, and low cost [23]. Most researches, such as in [23]-[25], are using features of cortical bone from dental panoramic radiographs for osteoporosis detection. Another research proposed the use of mandibular condyle for detection of temporo-mandibular joint (TMJ) dislocation and its abnormalities [26].
According to [27], teeth images from dental panoramic radiographs can be used for forensic age estimation. Observation of teeth on dental panoramic radiographs can also be used to measure the alveolar bone resorption, which is one of the most important sign of periodontal disease [28]. Fig. 1 shows an example of dental panoramic radiographs and also several objects on it such as the teeth, cortical bone, and mandibular condyle. Intensities of objects and background on dental panoramic radiographs are similar, hence segmentation based on intensity slicing is difficult to perform [28].

\section{Proposed Method}

Interactive segmentation using statistical analysis for region merging consist of four processes, which are region splitting, user marking, distance measurement, and region merging. The detail of this method is shown in Fig. 2.

In the region splitting process, mean-shift segmentation software provided by Edison System is used to create the initial segmented image [29]. The mean-shift algorithm split the input image based on probability density functions gradient of the image. Starting with the center of any cluster of pixels in an image, mean shift determine a center of mass as the point facing the densest region and then the center of the cluster is shifted to that mass. The process repeated until a zero vector is attained [30].

In this paper we focus on proposing a new distance measurement and region merging strategy. From the region splitting process, let the input image $(I)$ splitted into $K$ regions $[1,2, \ldots, K]$ which compose a single cluster namely non-marked cluster $(C)$ :

$$
I=C=\left\{C_{i}\right\}_{i=1, \ldots, K}
$$

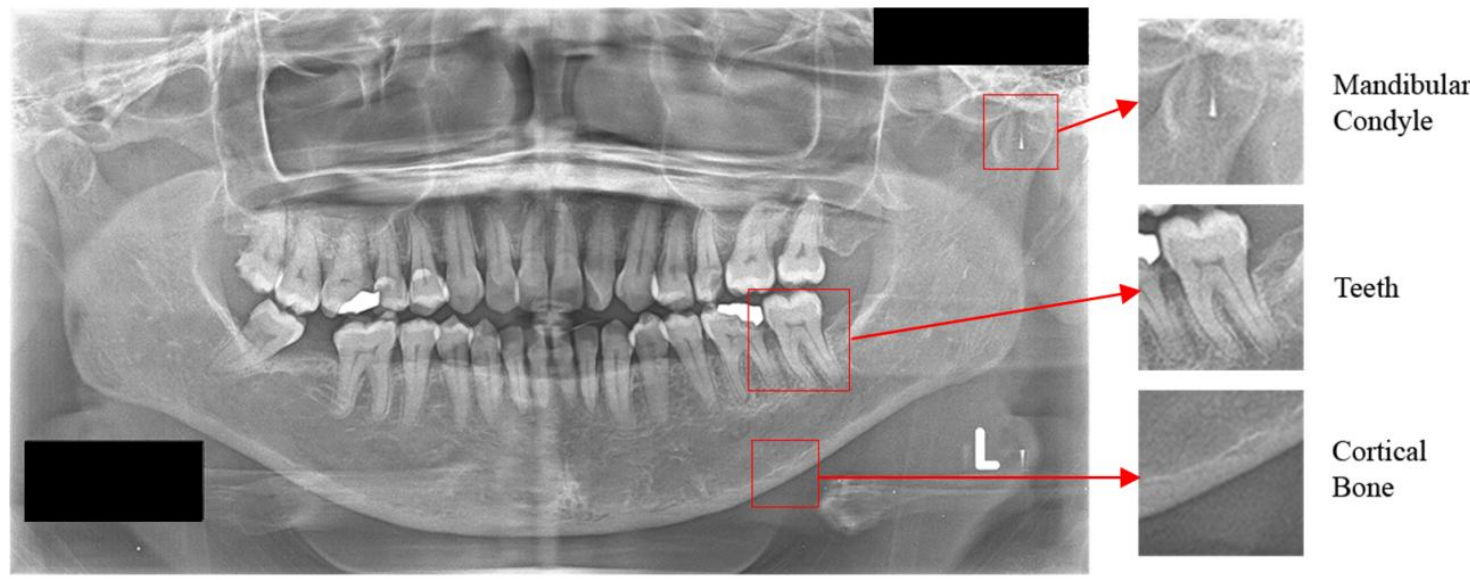

Fig. 1. Dental panoramic radiographs

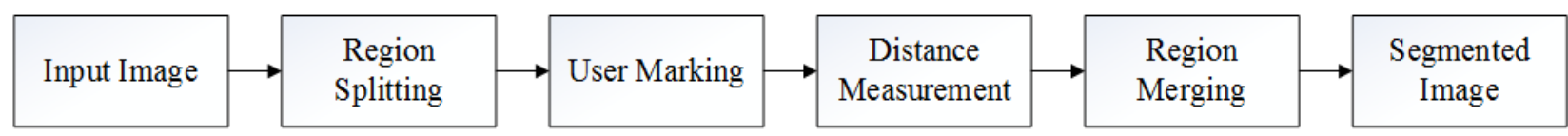

Fig. 2. Flowchart of the proposed method 


\section{III.1. User Marking}

In an interactive segmentation method, user need to give some information to the method about the criteria of the object and the background on the image. After get the initial segmented image using mean-shift algorithm, user needs to draw several object and background markers on the segmented image to specify whether a region is an object or background.

If a region $k$ is marked as an object, then the region will be moved to the object cluster $(O)$. If a region is marked as an background, then the region will be moved to the background cluster $(B)$. If a region is not marked as an object or background, the region will remain in the non-marked cluster $(C)$. The regions in the non-marked cluster will be moved to either object or background cluster on the next process.

Object cluster $(O)$ and background cluster $(B)$ consist of several regions so that:

$$
\begin{aligned}
& O=\left\{O_{i}\right\}_{i=1, \ldots, n} \\
& B=\left\{B_{i}\right\}_{i=1, \ldots, m}
\end{aligned}
$$

where $n$ is the number of regions that compose the object cluster and $m$ is the number of regions that compose the background cluster. Now the input image is consisted of three clusters namely object cluster $(O)$, background cluster $(B)$, and non-marked cluster $(C)$ :

$$
I=\{C, O, B\}
$$

\section{III.2. Distance Measurement}

After user defined the sample regions for object and background cluster, the characteristics or features difference between the non-marked cluster to each of the object and background cluster are measured. The resulting distance will be used as the criterion in the region merging strategy. The proposed distance measurement method use inter-class variance approach to calculate the distance between a non-marked region to either of object and background cluster.

\section{III.2.1. Features Extraction}

Before measuring the difference between features of the regions, the value of the features from each regions in the image must be obtained. Hence the features selection is an important thing. In this paper we provide two scenarios: for grayscale and color images. For grayscale images, such as dental panoramic radiographs, the extracted feature is the gray level of the pixels. For color images, the extracted feature is the HSI (hue, saturation, and intensity) values of the pixels.

HSI color space separates the color from the intensity so that the color and the intensity of the image can be compared separately and does not result in highly correlated channels [31]. Based on that consideration, features extracted on distance measurement process for color images are the hue, saturation, and intensity values of each regions in the image.

\section{III.2.2. Inter-class Variance}

Inter-class variance use the ratio of area between a region and the image, and the mean value of the pixels in a region. Let the given imge separated into $K$ regions. The number of the pixels in region $k$ is denoted by $n_{k}$ and the total number of the pixels in the given image is denoted by $N=n_{1}+n_{2}+\ldots+n_{K}$. Let the ratio of the area between region $k$ with $n_{k}$ pixels and the image with $N$ pixels is denoted by $\omega_{k}$ then:

$$
\omega_{k}=\frac{n_{k}}{N}
$$

Let the pixel values of region $k$ be represented in $L_{k}$ levels $\left[1,2, \ldots, L_{k}\right]$ and the number of pixels in region $k$ at level $i$ is denoted by $x_{i}$. Then the number of pixels in the region $k$ is $n_{k}=x_{1}+x_{2}+\ldots+x_{L_{k}}$. Let the mean value of the pixels in region $k$ is denoted by $\mu_{k}$ then:

$$
\mu_{k}=\frac{\sum_{i=1}^{L_{k}} x_{i}}{n_{k}}
$$

Inter-class variance $\sigma_{I}^{2}$ between two clusters is the sum of the square distances between the means of the two clusters and the total mean of both clusters $\mu_{T}$, as defined in (7) [9]. Using (7-9) it will be easily verified that the inter-class variance $\sigma_{I}^{2}$ between two clusters can be obtained as in (10). Using the same viewpoint as in (10), the inter-class variance among $n$ clusters can be written as in (11):

$$
\begin{gathered}
\sigma_{I}^{2}=\omega_{1}\left(\mu_{1}-\mu_{T}\right)^{2}+\omega_{2}\left(\mu_{2}-\mu_{T}\right)^{2} \\
\omega_{1} \mu_{1}+\omega_{2} \mu_{2}=\mu_{T} \\
\omega_{1}+\omega_{2}=1 \\
\sigma_{I}^{2}=\omega_{1} \omega_{2}\left(\mu_{2}-\mu_{1}\right)^{2} \\
\sigma_{I}^{2}=\sum_{i=1}^{n} \sum_{j=1}^{n} \omega_{i} \omega_{j}\left(\mu_{j}-\mu_{i}\right)^{2}
\end{gathered}
$$

\section{III.2.3. Distance Measurement on Inter-class Variance}

Because the proposed method needs to calculate the inter-class variance between a region and a cluster (not among clusters), the variance among regions in the same cluster do not need to be calculated. Let the given region defined as $k$ and the given cluster $V$ consists of $n$ regions. Based on (11), the inter-class variance between a single region $k$ and cluster $V$ can be obtained using the proposed formula in (12): 


$$
\sigma_{I}^{2}=\sum_{i=1}^{n} \omega_{k} \omega_{V i}\left(\mu_{V i}-\mu_{k}\right)^{2}
$$

Distance measurement between a region in the nonmarked cluster and the either object cluster or background cluster is done using inter-class variance. Let the object cluster $O$ consist of $n$ regions $\left[O_{1}, O_{2}, \ldots, O_{n}\right]$ and the background cluster $B$ consist of $m$ regions $\left[B_{1}, B_{2}, \ldots, B_{m}\right]$. Based on (12), the distance Dist ${ }_{\text {ock }}$ between region $C_{k}$ and the object cluster $O$ can be written as in (13) and the distance Dist ${ }_{B C k}$ between region $C_{k}$ to the background cluster $B$ can be written as in (14), where $\omega_{C k}$ is the area ratio of the region $C_{k}, \omega_{O i}$ is the area ratio of the region in the object cluster $O_{i}$, and $\omega_{B i}$ is the area ratio of the region in the background cluster $B_{i}$ :

$$
\begin{aligned}
\operatorname{Dist}_{O C_{k}} & =\sum_{i=1}^{n} \omega_{C_{k}} \omega_{O_{i}}\left(\mu_{O_{i}}-\mu_{C_{k}}\right)^{2} \\
\operatorname{Dist}_{B C_{k}} & =\sum_{i=1}^{m} \omega_{C_{k}} \omega_{B_{i}}\left(\mu_{B_{i}}-\mu_{C_{k}}\right)^{2}
\end{aligned}
$$

Formula in (13) and (14) can be used to calculate the distance from a region to object and background clusters respectively for grayscale images. For color images, since we used HSI features then $\mu$ is a vector that consist of three features $\mu_{1}, \mu_{2}$, and $\mu_{3}$ correspond to hue, saturation, and intensity mean values, respectively. Using Euclidean distance, the distance measurement formula in (13) and (14) can be written as in (15) and (16):

$$
\begin{aligned}
\operatorname{Dist}_{O C_{k}} & =\sum_{i=1}^{n} \omega_{C_{k}} \omega_{O_{i}}\left\|\mu_{O_{i}}-\mu_{C_{k}}\right\| \\
\operatorname{Dist}_{B C_{k}} & =\sum_{i=1}^{m} \omega_{C_{k}} \omega_{B_{i}}\left\|\mu_{B_{i}}-\mu_{C_{k}}\right\|
\end{aligned}
$$

\section{III.3. Region Merging}

The purpose of region merging process is to merge a region in the non-marked cluster into object or background cluster repeatedly until the non-marked cluster is empty. The idea is to find the non-marked region which is most similar to the clusters and merge it. The similarity criteria has been obtained in the distance measurement process.

A non-marked region is merged into either object or background if it has the minimal distance value compared to all of the non-marked regions. After get the distance between each region in non-marked cluster to the background and object cluster, region merging process is done by finding the region with minimal distance value $\left(t^{*}\right)$. First, find the region with minimal distance to object cluster $t_{O}{ }^{*}$ and the region with minimal distance to background cluster $t_{B}{ }^{*}$. Those distances are compared to find which region has smaller distance value $C_{t^{*}}$ :

$$
\begin{gathered}
t_{O}{ }^{*}=\arg \min _{1 \leq k \leq K} \text { Dist }_{O C_{k}} \\
t_{B}{ }^{*}=\arg \min _{1 \leq k \leq K} \text { Dist }_{B C_{k}} \\
t^{*}=\arg \min \left(\text { Dist }_{t_{O}{ }^{*}} \text { Dist }_{t_{B}}\right)
\end{gathered}
$$

Region with minimal distance value $C_{t^{*}}$ is merged into either object or background cluster, depends on its distance. If its distance to object cluster Dist $_{t_{O}}{ }^{*}$ is smaller than its distance to background cluster Dist $_{t_{B}}{ }^{*}$ then the region is merged to the object cluster, and vice versa:

$$
\begin{aligned}
& O= \begin{cases}O & \text { if } \text { Dist }_{t_{B}} \leq \text { Dist }_{t_{O^{*}}} \\
O \cup C_{t^{*}} & \text { if } \text { Dist }_{t_{B}}>\text { Dist }_{t_{O^{*}}}\end{cases} \\
& B= \begin{cases}B & \text { if Dist } t_{B_{B}}>\text { Dist }_{t_{O^{*}}} \\
B \cup C_{t^{*}} & \text { if Dist } t_{t_{B}} \leq \text { Dist }_{t_{O^{*}}}\end{cases}
\end{aligned}
$$

After region with minimal distance is merged, the distance measurement and region merging process are repeated to the remaining regions in the non-marked cluster until the non-marked cluster is empty. The result is an image that consists only of object and the background cluster. Image which object and background has been separated is called segmented image:

$$
I=\{O, B\}
$$

\section{Result and Discussion}

In order to evaluate its performance, the proposed method has been tested using two grayscale images dan four color images (see Figs. 3). The corresponding ground truth segmentations of the test images are shown in Figs. 4. The color images (a) - (d) and its ground truth has been provided by Weizmann's segmentation evaluation database which represent natural images [32]. The grayscale image (e) with uneven exposure is obtained from Matlab image dataset [20].

The proposed region merging strategy used especially for low contrast image such as dental panoramic radiographs. Hence we used several images obtained from dental panoramic radiographs containing several objects on dental panoramic radiographs. The dataset of dental panoramic radiographs is obtained from UNAIR Hospital. Our experiment used 7 images of ROIs (region of interest) obtained from dental panoramic radiographs which consist of 2 teeth images (f) - (g), 2 cortical bone images (h) - (i), and 3 mandibular condyle images (j) (1).

We have compared the proposed method with Extracted Pixels Similarity Features (EPSF) in [20] that is an interactive segmentation method using maximal similarity based region merging (MSRM) method and 
Otsu thresholding method that has been widely used in automatic image segmentation and become the base of much development in segmentation method using statistical analysis. Figs. 5 show the splitted regions by Edison System and the markers given by user for the proposed method in Figs. 6 and for the EPSF method in Figs. 7. Lines with red color mark the object regions and lines with blue color mark the background regions. The segmentation results of the proposed method, EPSF, and Otsu thresholding are shown in Figs. 6-8, respectively. Those results are evaluated using Misclassification Error (ME) and Relative Foreground Area Error (RAE) [8].

ME calculates the ratio of object pixels that wrongly classified as background, and vice versa. ME can be expressed as:

$$
M E=1-\frac{\left|O_{g} \cap O_{r}\right|+\left|B_{g} \cap B_{r}\right|}{\left|O_{g}\right|+\left|B_{g}\right|}
$$

where $O_{g}$ and $B_{g}$ is the object and background pixels in the ground truth image and $O_{r}$ and $B_{r}$ is the object dan background pixels in the segmentation result. RAE measures the ratio of the difference between object's area in ground truth image and the segmentation result. RAE can be written as:

$$
R A E= \begin{cases}\frac{A_{g}-A_{r}}{A_{g}} & \text { if } A_{r}<A_{g} \\ \frac{A_{r}-A_{g}}{A_{r}} & \text { if } A_{r} \geq A_{g}\end{cases}
$$

where $A_{g}$ is area of the object in the ground truth image and $A_{r}$ is the area of the object in the segmentation result. The range of ME and RAE value are between 0 and 1. If the ME and RAE value is 0 then the segmentation result is exactly the same as the ground truth image.

Table II shows the ME and RAE values of the segmentation results of the proposed method and the comparison methods compared with the segmentation ground truth images. The average ME value of the proposed method, EPSF method, and Otsu thresholding are $1.47 \%, 6.02 \%$, and $5.54 \%$ respectively. The average RAE value of the proposed method, EPSF method, and
Otsu thresholding are $8.12 \%, 26.74 \%$, and $22.64 \%$ respectively.

Although, many automatic segmentation methods have been developed, the manual segmentation process by the user is still necessary to derive the system to correctly classify the object in a natural image. If the segmentation process is done automatically then the result will not considering the user's need. But if the segmentation process is done manually, then the problem of subjectivity will arise. Hence the integration between automatic and manual segmentation, which is called semi-automatic segmentation, are needed so that the segmentation process can be done objectively but still consider the user's need by using the representative sample from the user.

From the experimental results, some points can be discussed about the proposed method and the interactive image segmentation in general. We use EPSF in our experiment for comparison method because EPSF method using maximal similarity based region merging (MSRM) that use adjacency feature in region merging process. The example of automatic segmentation for comparison between automatic and interactive segmentation method is Otsu thresholding because Otsu thresholding use statistical analysis with inter-class variance to separate object and background cluster.

Image (a) - (d) are basically a simple natural image where the object and the background of the images are clearly visible so that automatic segmentation like Otsu thresholding can give accurate segmentation results in general, except in image (c) where the color of object and the background is slightly similar so that Otsu thresholding failed to give accurate segmentation result. In image (a) and (b), the segmentation results of the proposed method is better than the EPSF because the proposed method considers the proportion of a region with the image so that it can separate a small object, which is the airplane's propeller, with its background.

In image (c), EPSF failed to separate the airplane's tail with its background because MSRM method that is used in region merging process of EPSF uses adjacency to merge a region with the background cluster, so that the background between airplane's body and tail is wrongly identified as object. In image (d), both of the proposed and EPSF method give an accurate segmentation results.

TABLE II

\begin{tabular}{|c|c|c|c|c|c|c|}
\hline \multirow{2}{*}{ Image } & \multicolumn{2}{|c|}{ Proposed Method } & \multicolumn{2}{|c|}{ EPSF Method } & \multicolumn{2}{|c|}{ Otsu Thresholding } \\
\hline & $\operatorname{ME~(\% )}$ & RAE (\%) & $\mathrm{ME}(\%)$ & RAE (\%) & ME (\%) & RAE (\%) \\
\hline$a$ & 1.41 & 17.28 & 1.68 & 19.27 & 0.96 & 11.05 \\
\hline$b$ & 1.13 & 6.15 & 2.25 & 26.49 & 1.56 & 16.85 \\
\hline$c$ & 1.95 & 13.87 & 2.34 & 10.16 & 8.68 & 63.04 \\
\hline$d$ & 1.05 & 3.76 & 1.89 & 4.79 & 4.46 & 18.55 \\
\hline$e$ & 0.72 & 1.66 & 26.27 & 95.58 & 2.98 & 4.96 \\
\hline$f$ & 3.72 & 4.29 & 3.61 & 4.61 & 16.26 & 15.64 \\
\hline$g$ & 6.47 & 9.21 & 9.23 & 1.36 & 10.39 & 7.25 \\
\hline$h$ & 2.27 & 6.49 & 2.21 & 3.32 & 45.86 & 63.23 \\
\hline$i$ & 1.55 & 3.12 & 2.64 & 6.57 & 37.95 & 56.58 \\
\hline$j$ & 1.37 & 1.56 & 3.02 & 5.25 & 46.60 & 21.81 \\
\hline$k$ & 3.30 & 3.34 & 10.02 & 12.96 & 61.13 & 64.66 \\
\hline$l$ & 2.45 & 1.73 & 4.21 & 4.85 & 58.04 & 59.00 \\
\hline
\end{tabular}

Performance Evaluation of The Proposed Method COMPAREd to EPSF Method AND OtSU THRESHOLDING 


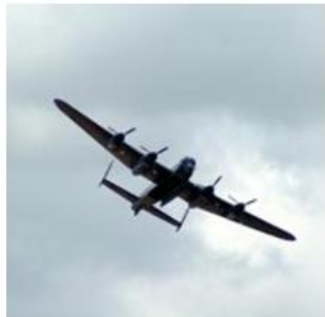

(a)

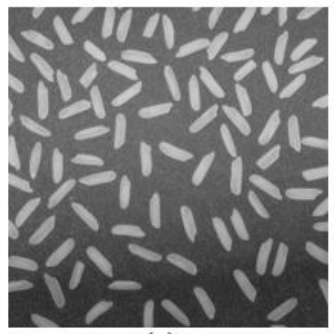

(e)

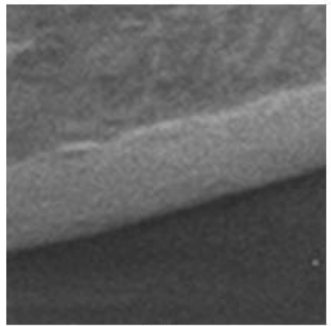

(i)

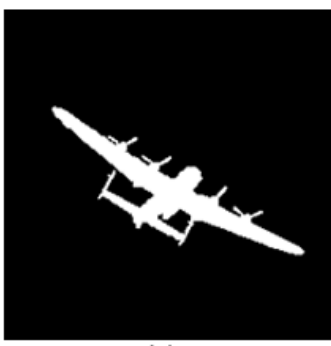

(a)

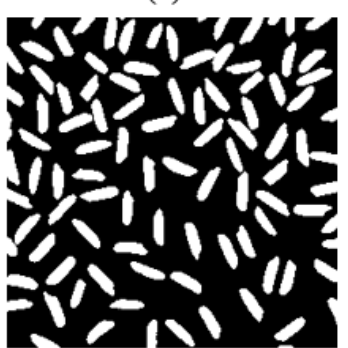

(e)

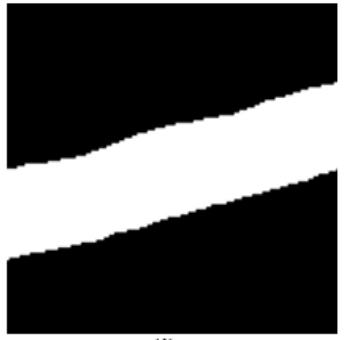

(i)

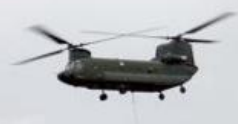

(b)

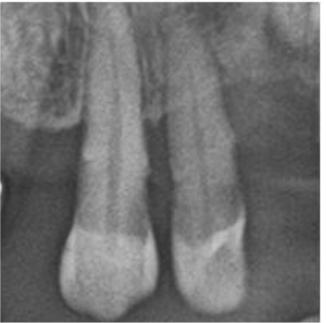

(f)

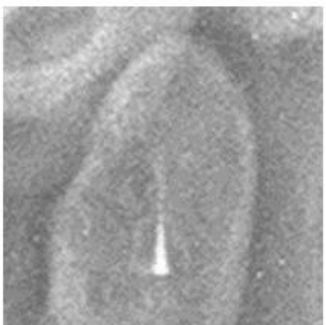

(j)

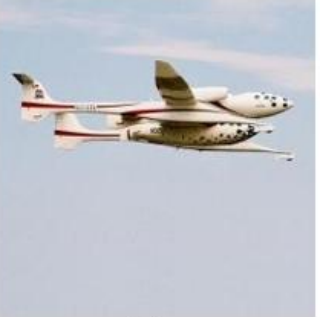

(c)

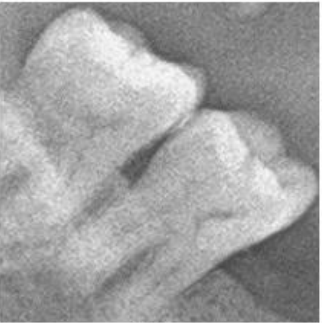

(g)

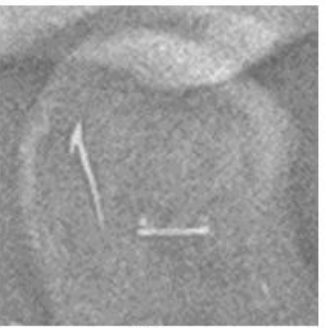

(k)

Figs. 3. Input images

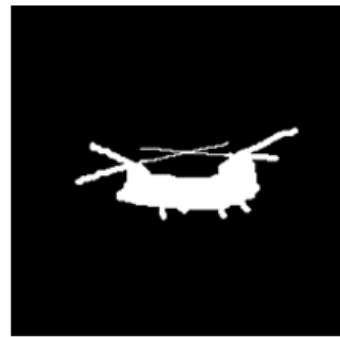

(b)

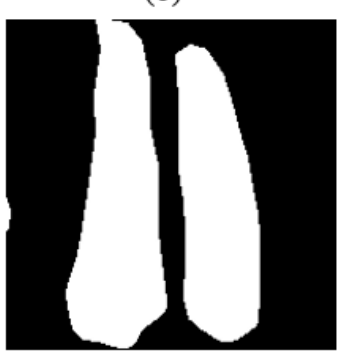

(f)

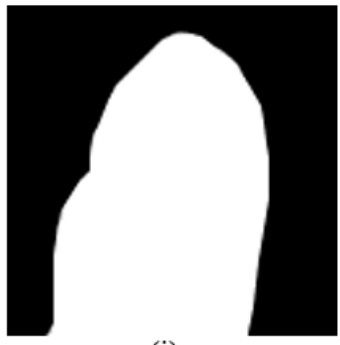

(j)

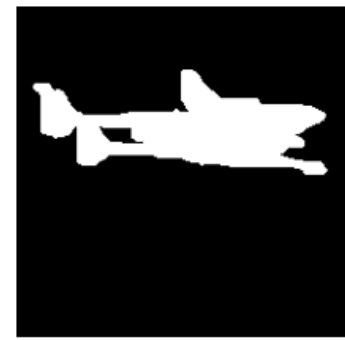

(c)

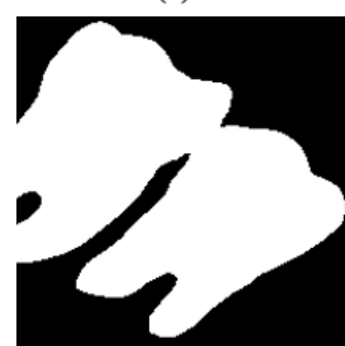

(g)

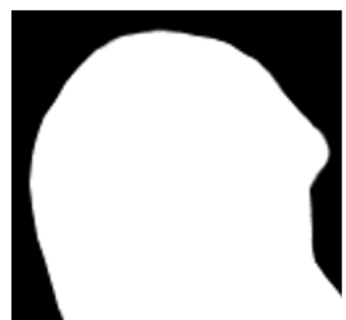

(k)

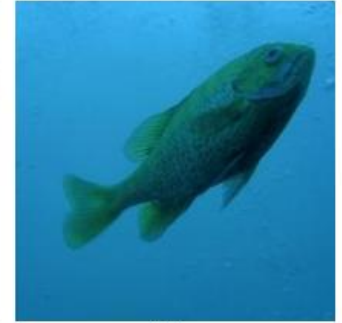

(d)

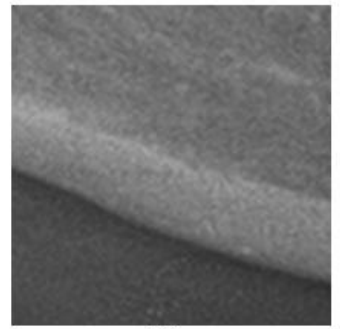

(h)

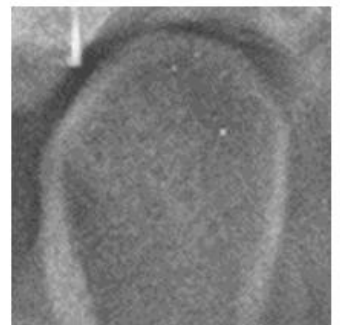

(1)

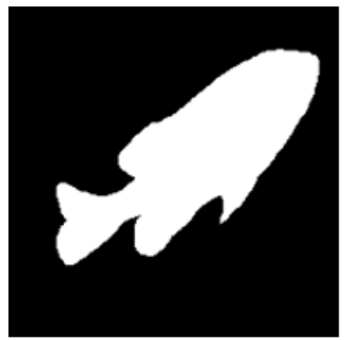

(d)

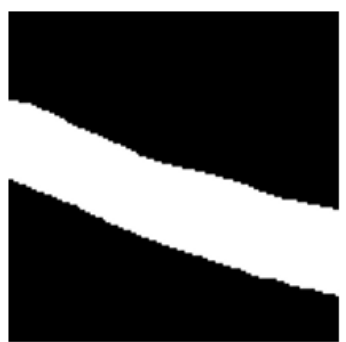

(h)

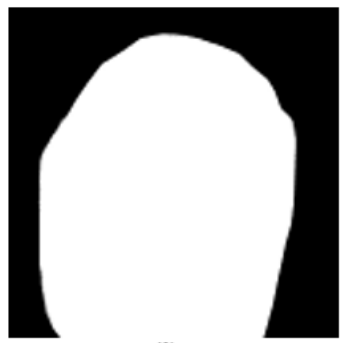

(1)

Figs. 4. Ground truth images 


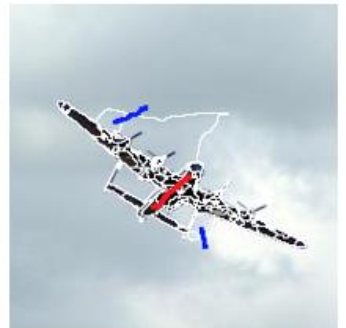

(a)

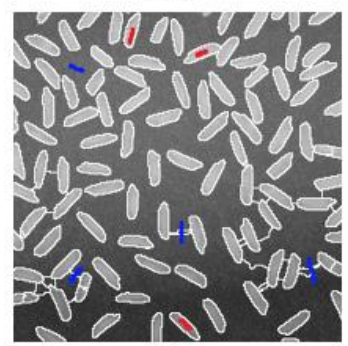

(e)

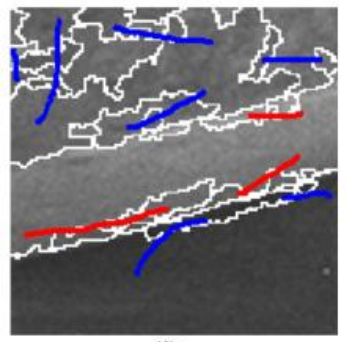

(i)

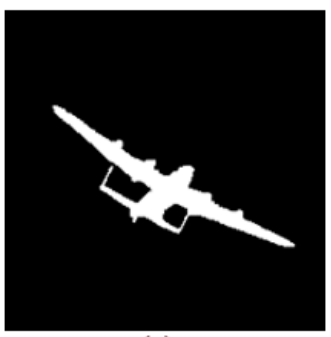

(a)

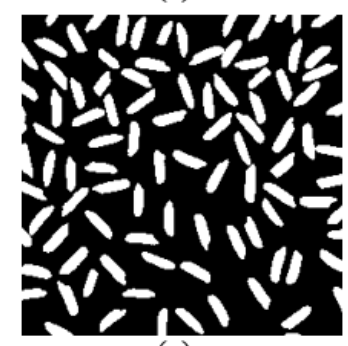

(e)

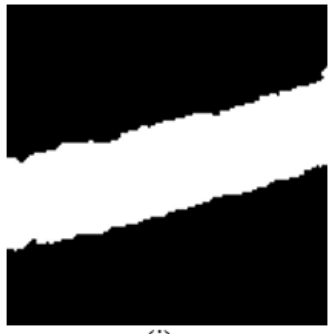

(i)

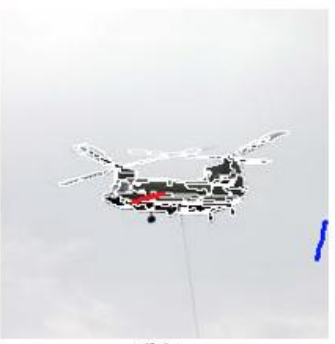

(b)

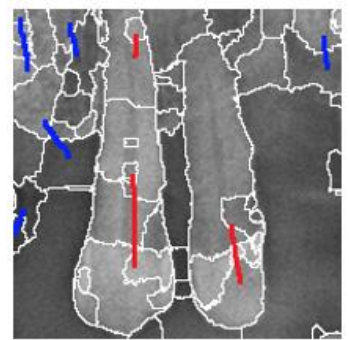

(f)

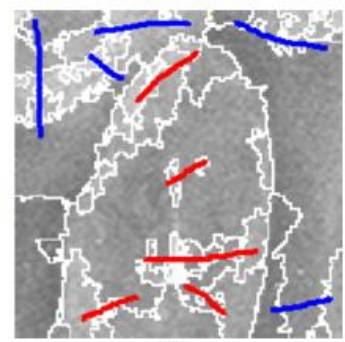

(j)

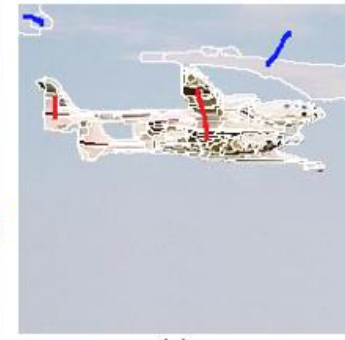

(c)

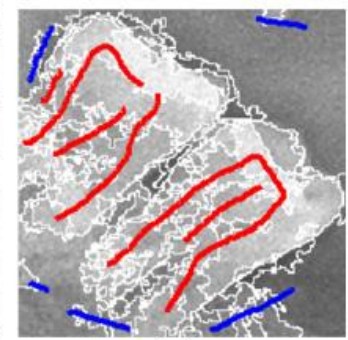

(g)

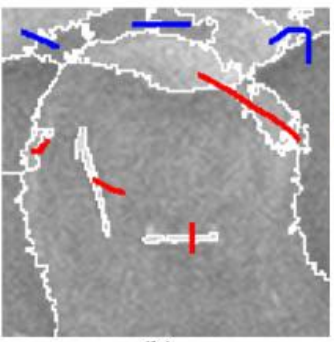

(k)

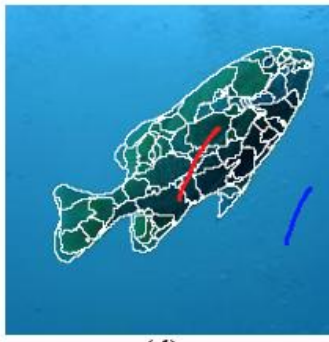

(d)

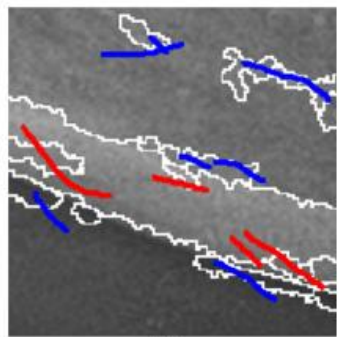

(h)

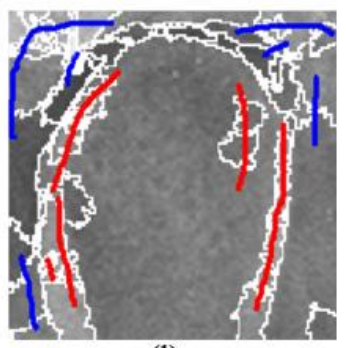

(1)

Figs. 5. Splitted regions by Edison System and its markers

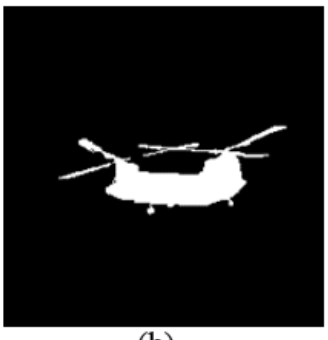

(b)

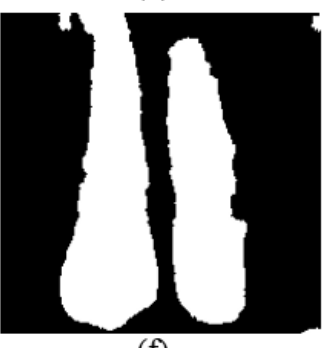

(f)

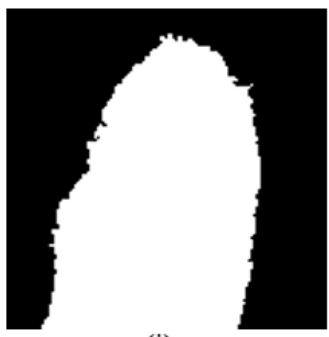

(j)

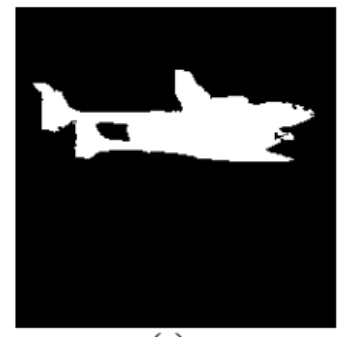

(c)

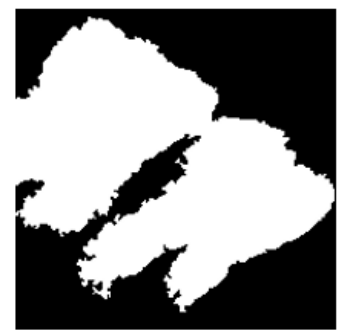

(g)

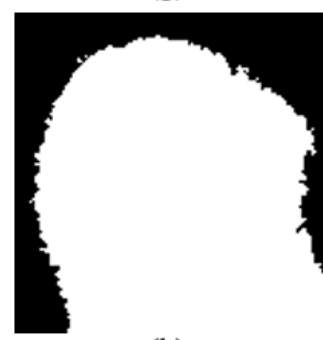

(k)

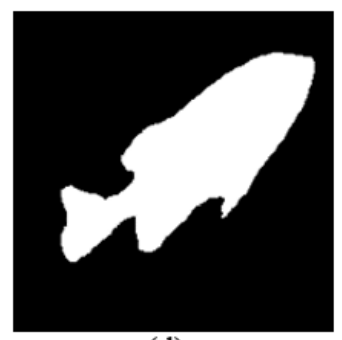

(d)

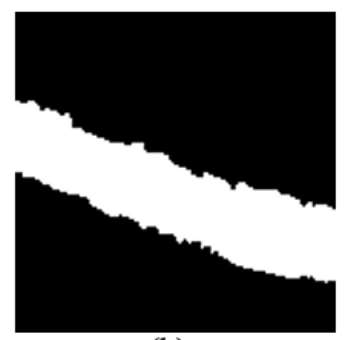

(h)

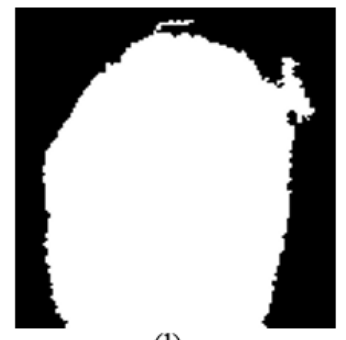

(1)

Figs. 6. Segmentation results of the proposed method 


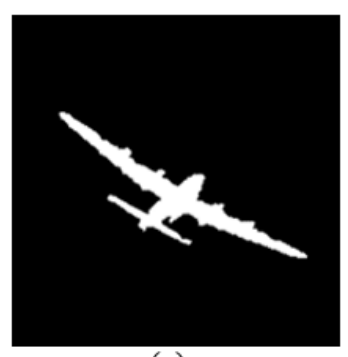

(a)

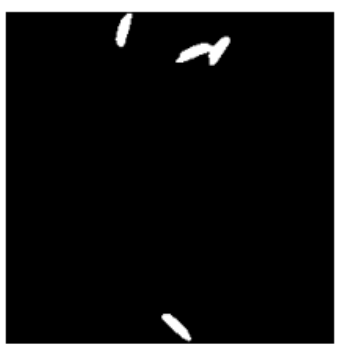

(e)

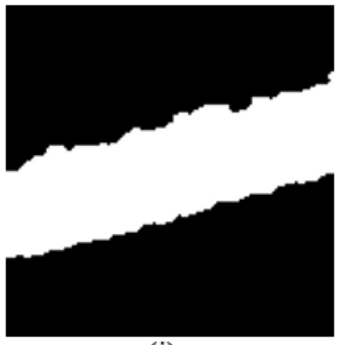

(i)

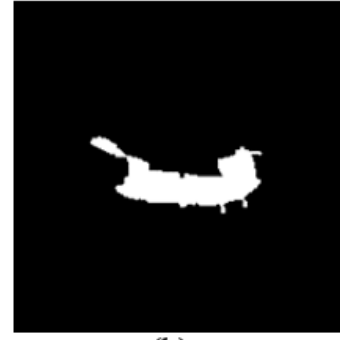

(b)

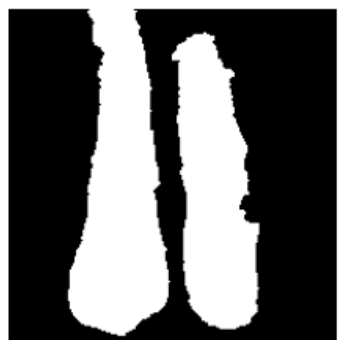

(f)

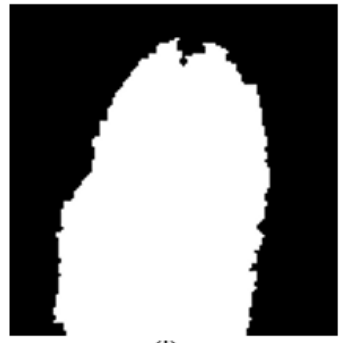

(i)

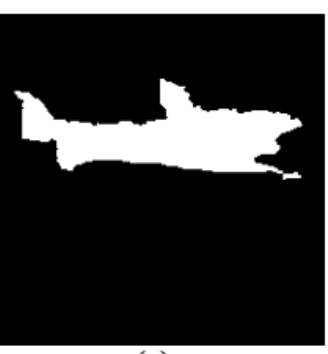

(c)

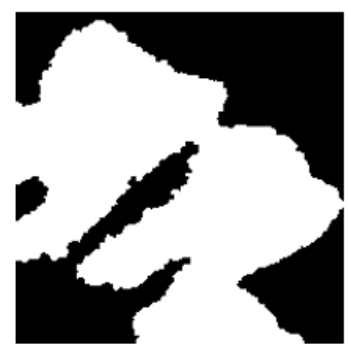

(g)

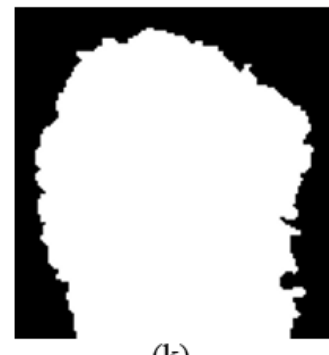

(k)

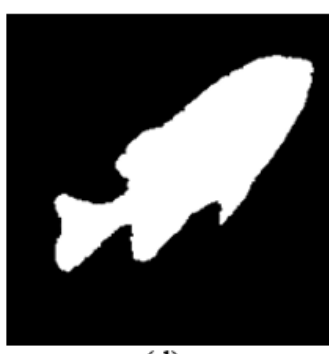

(d)

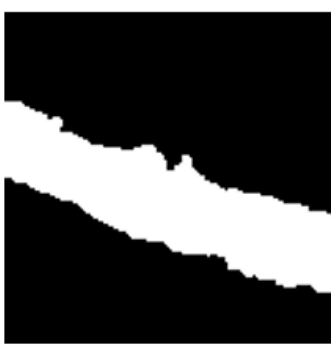

(h)

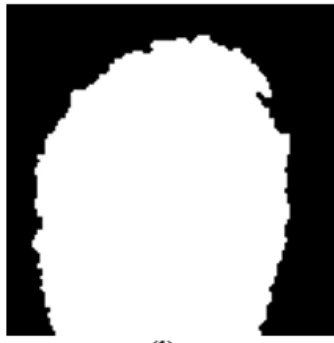

(1)

Figs. 7. Segmentation results of EPSF method

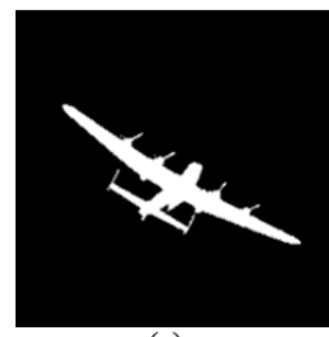

(a)

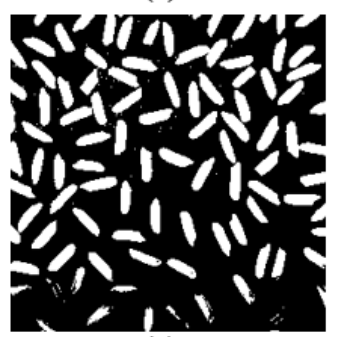

(e)
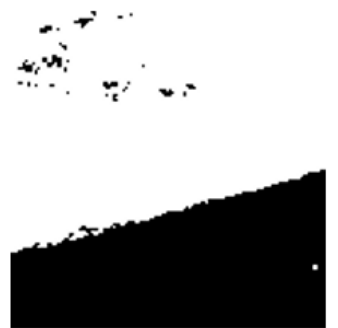

(i)

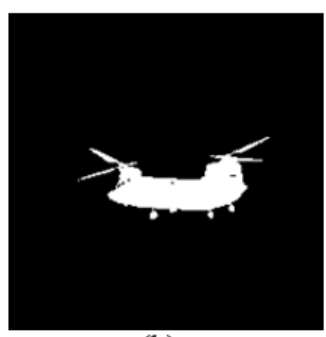

(b)

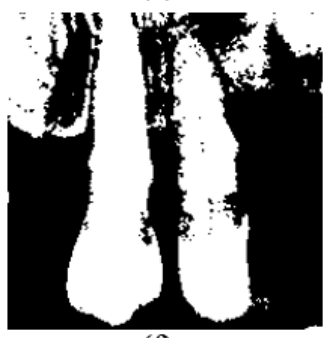

(f)

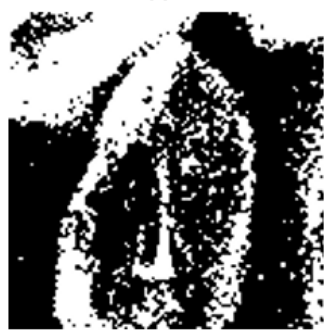

(j)

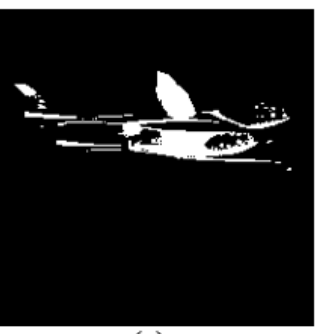

(c)

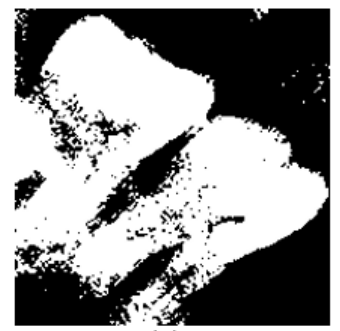

(g)

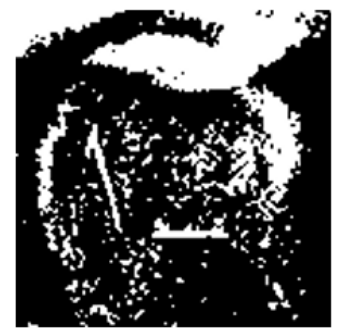

(k)

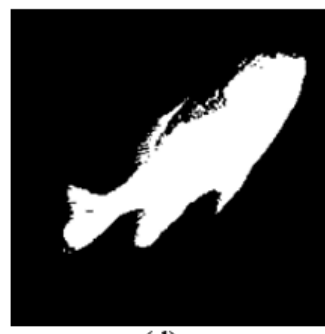

(d)

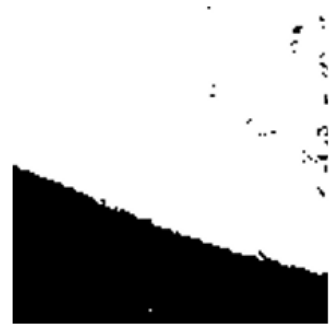

(h)

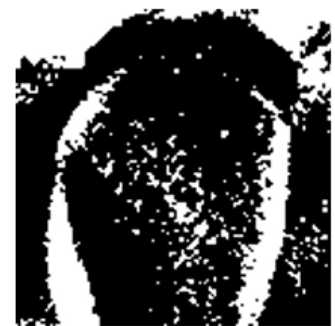

(1)

Figs. 8. Segmentation results of Otsu thresholding 
Image (e) is basically not a complex image, but image (e) has an uneven exposure so that automatic image segmentation will need a preprocessing to enhance the image so that the image will have an even exposure. Using the proposed method, the enhancement process is not needed because user can give several samples of object and background regions to the system so that the system "know" the range of gray level of the object and background regions in the image. The EPSF method did not give an accurate segmentation result because the uneven exposure make the method "confused" when measured the distance between regions using color and texture features. Since EPSF method will merge every adjacent region with background samples as background cluster, then all of the regions except the regions that marked as object will be identified as background.

The number and the significance of the samples that taken by user affects the segmentation result. For example, if in image (e) user only gives sample of the object in regions with high exposure then the object with low exposure can be classified as background. Also, greater number of samples will not always gives a better segmentation result if the samples is not balanced. For example, if in image (e) user gives sample of the object with both low and high exposure regions but the number of marked high exposure regions is significantly greater than the number of marked low exposure regions then the proposed method will associate the object with mostly high exposure region so that several object regions with low exposure will be incorrectly classified as the background. Hence, is recommended that the user gives an adequate number of object and background samples which are representative.

An example of low contrast images from dental panoramic radiographs has been provided by image (f) (l) where the gray level of the object and the background is quite similar so that the automatic segmentation cannot separate the image and the background accurately. Using interactive image segmentation, user can provide a number of samples of object and background regions in the image so that the segmentation process is done based on the samples. Although the object and the background in the image are slightly different, but the presence of the samples from the user will give some reference to the methods so that the error of segmentation result from both of the interactive segmentation method, which are the proposed method and the EPSF method, are lower than segmentation error of Otsu thresholding.

The proposed method is not using adjacency feature of a region to do region merging process because if there are any background regions which are separated completely by the object and did not marked by the user, then those background regions will be labeled as object as shown in image (c) where the EPSF method cannot identify the background between the airplane's body and tail. In general, the proposed method will give much lower segmentation error than EPSF and Otsu thresholding method especially on image with uneven exposure and low contrast.

\section{Conclusion}

Interactive image segmentation that integrates automatic and manual segmentation process is needed so that the segmentation process can be done objectively but still consider the user's need by using the representative sample from the user. The proposed method uses a new distance measurement using discriminant analysis for region merging strategy that refers to the most representative sample regions which are selected by user so that the similarity between region and the representative regions will still be preserved.

The experimental results show that interactive image segmentation can give a higher segmentation accuracy than automatic segmentation method. And in several type of images, the proposed region merging strategy using inter-class variance to measure the distance between regions has proven to give a high segmentation accuracy both for natural and low contrast images.

Based on the experimental results, the proposed method can be considered as appropriate segmentation method for dental panoramic radiographs and other medical images that have low contrast. To improve the accuracy of segmentation results of the proposed method, combination with other methods such as morphological operations can be used. However, further research must be done to develop method for region splitting process that can provide regions with smooth boundary in low contrast images. For future works, analysis of features selection that will be used for measure the distance between regions for low contrast and grayscale images such as dental panoramic radiographs can be done.

\section{References}

[1] J. Ning, L. Zhang, D. Zhang, C. Wu, Interactive image segmentation by maximal similarity based region merging, (2010) Pattern Recognition, 43, pp. 445-456.

http://dx.doi.org/10.1016/j.patcog.2009.03.004

[2] G. Chandramohan, S. Subramanian, An Efficient Hybrid Segmentation Algorithm for Computer Tomography Image Segmentation, (2014) International Review on Computers and Software (IRECOS), 9 (9), pp. 1576-1582. https://doi.org/10.15866/irecos.v9i9.3039

[3] T. N. A. Nguyen, J. Cai, J. Zheng, J. Li, Interactive object segmentation from multi-view images, (2013) Journal of Visual Communication and Image Representation, 24 (4), pp. 477-485. http://dx.doi.org/10.1016/j.jvcir.2013.02.012

[4] Y. Attaf, A. Adane, M. Lahdir, A. Boudraa, M. Laghrouche, Z. Ameur, An AM-FM Based Image Segmentation: Detection of Clouds in MSG Images of Algeria, (2015) International Review on Computers and Software (IRECOS), 10 (7), pp. 789-797. https://doi.org/10.15866/irecos.v10i7.7107

[5] T. Li, Z. Xie, J. Wu, J. Yan, L. Shen, Interactive object extraction by merging regions with k-global maximal similarity, (2013) Neurocomputing, 120 , pp. 610-623. http://dx.doi.org/10.1016/j.neucom.2013.04.009

[6] Lakshmi, M., Prasad, S., Rahman, M., Efficient Speckle Noise Reduction Techniques for Synthetic Aperture Radars in Remote Sensing Applications, (2016) International Review of Aerospace Engineering (IREASE), 9 (4), pp. 114-122. https://doi.org/10.15866/irease.v9i4.10367

[7] Y. Yang, S. Han, T. Wang, W. Tao, X.-C. Tai, Multilayer graph cuts based unsupervised color-texture image segmentation using multivariate mixed student's t-distribution and regional credibility merging, (2013) Pattern Recognition, 46, pp. 1101-1124. 
http://dx.doi.org/10.1016/j.patcog.2012.09.024

[8] A. Z. Arifin, A. Asano, Image Segmentation by Histogram Thresholding Using Hierarchical Cluster Analysis, (2006) Pattern Recognition Letters, 27 (13), pp. 1515-1521. http://dx.doi.org/10.1016/j.patrec.2006.02.022

[9] N. Otsu, A threshold selection method from gray-level histograms, (1979) IEEE Transactions of Systems, Man, and Cybernetics, (9), pp. 62-66. http://dx.doi.org/10.1109/TSMC.1979.4310076

[10] K. McGuinness, N. E. O'Connor, A comparative evaluation of interactive segmentation algorithms, (2010) Pattern Recognition, 43, pp. 434-444. http://dx.doi.org/10.1016/j.patcog.2009.03.008

[11] R. Adams, L. Bischof, Seeded Region Growing,(1994) IEEE Transactions on Pattern Analysis and Machine Intelligence, 16 (6), pp. 641-647. http://dx.doi.org/10.1109/34.295913

[12] M. Eapen, R. Korah, Integration of Improved Region Growing (iRG) and Level Set Method for Automated Medical Image Segmentation, (2014) International Review on Computers and Software (IRECOS), 9 (2), pp. 278-284.

[13] M. Kass, A. Witkin, D. Terzopoulos, Snakes: Active Contour Models, (1988) International Journal of Computer Vision, 1, pp. 321-331. http://dx.doi.org/10.1007/BF00133570

[14] B. Peng, L. Zhang, D. Zhang, J. Yang, Image segmentation by iterated region merging with localized graph cuts, (2011) Pattern Recognition, 44, pp. 2527-2538. http://dx.doi.org/10.1016/j.patcog.2011.03.024

[15] Mo Yan, P.-L. Shui, Interactive Image Segmentation Based on Gaussian Mixture Models with Spatial Prior, (2015) International Journal of Multimedia and Ubiquitous Engineering, 10 (7), pp. 105-114. http://dx.doi.org/10.14257/ijmue.2015.10.7.11

[16] E. Zemene, M. Pelillo, Interactive Image Segmentation Using Constrained Dominant Sets, in European Conference on Computer Vision. Springer International Publishing, 2016. http://dx.doi.org/10.1007/978-3-319-46484-8_17

[17] P. Salembier, L. Garrido, Binary partition tree as an efficient representation for image processing, segmentation, and information retrieval, (2000) IEEE Transactions on Image Processing, 9, pp. 561-576. http://dx.doi.org/10.1109/83.841934

[18] T. Adamek, Using contour information and segmentation for object registration, modeling and retrieval, Dublin City University: Ph.D. Dissertation, 2006.

[19] X. H. Zeng, R. H. Yi, S. W. Zhu, S. S. He, Auto-marking Image Segmentation Based Manifold Ranking, in International Conference on Artificial Intelligence and Industrial Engineering (AIIE), 2015.

[20] A. S. Sankoh, A. Z. Arifin, A. Y. Wijaya, Extracted Pixels Similarity Features (EPSF) using Interactive Image Segmentation Techniques, (2016) International Journal of Computer Applications, 136 (2), pp. 5-12. http://dx.doi.org/10.5120/ijca2016908236

[21] R. S. Basuki, M. Hariadi, E. M. Yuniarno, M. H. Purnomo, Spectral-Based Temporal-Constraint Estimation for SemiAutomatic Video Object Segmentation, (2015) International Review on Computers and Software (IRECOS), 10 (9), pp. 959965. https://doi.org/10.15866/irecos.v10i9.7542

[22] N. Shah, N. Bansal, A. Logani, Recent advances in imaging technologies in dentistry, (2014) World Journal of Radiology, 6 (10), pp. 794-807. http://dx.doi.org/10.4329/wjr.v6.i10.794

[23] S. Geary, F. Selvi, S.-K. Chuang, M. August, Identifying Dental Panoramic Radiograps Features for the Screening of Low Bone Mass in Postmenopausal Women, (2015) International Journal of Oral and Maxillofacial Surgery, 44, pp. 395-399. http://dx.doi.org/10.1016/j.ijom.2014.11.008

[24] K. Horiba, C. Muramatsu, T. Hayashi, T. Fukui, T. Hara, A. Katsumata, H. Fujita, Automated Classification of Mandibular Cortical Bone on Dental Panoramic Radiographs for Early Detection of Osteoporosis, (2015) SPIE Medical Imaging, pp.
94142J-94142J, International Society for Optics and Photonics. http://dx.doi.org/10.1117/12.2081512

[25] M. S. Kavitha, S.-Y. An, C.-H. An, K.-H. Huh, W.-J. Yi, M.-S. Heo, S.-S. Lee, S.-C. Choi, Texture Analysis of mandibular cortical bone on digital dental panoramic radiographs for the diagnosis of osteoporosis in Korean women, (2015) Oral surgery, oral medicine, oral pathology and oral radiologi, 119 (3), pp. 346-356. http://dx.doi.org/10.1016/j.oooo.2014.11.009

[26] P. H. Shah, R. Venkatesh, Pulp/Tooth Ratio of Mandibular First and Second Molars on Panoramic Radiographs: An Aid for Forensic Age Estimation, (2016) Journal of Forensic Dental Sciences, 8 (2), pp. 112-115 http://dx.doi.org/10.4103/0975-1475.186374

[27] C, Muramtsu, R. Takahashi, R. Hayashi, T. Hara, T. Fukui, A. Katsumata, H. Fujita, Quantitative Evaluation of Alveolar Bone Resorption on Dental Panoramic Radiographs by Standardized Dentition Image Transformation and Probability Estimation, in Engineering in Medicine and Biology Society (EMBC), 2016 IEEE $38^{\text {th }}$ Annual International Conference, pp. 1038-1041, IEEE, 2016.

http://dx.doi.org/10.1109/EMBC.2016.7590880

[28] A. Naik, S. Tikhe, S. Bhide, K. P. Kaliyamurthie, T. Saravanan, Automatic Segmentation of Lower Jaw and Mandibular Bone in Digital Dental Panoramic Radiographs, (2016) Indian Journal of Science and Technology, 9 (11). http://dx.doi.org/10.17485/ijst/2016/v9i21/90293

[29] "Edison software," [Online]. Available: http://www.caip. rutgers.edu/riul/research/code.html.

[30] F. S. Kazi, I. A. Rizvi, M. M. Kadam, Segmentation using mean shift algorithm with modifications: A review, (2015) International Journal of Technical Research and Applications, 31, pp. 301-304.

[31] I. Omer, M. Werman, Color lines: image specific color representation, in Computer Vision and Pattern Recognition. CVPR 2004. Proceedings of the 2004 IEEE Computer Society Conference on Vol. 2, 2004 http://dx.doi.org/10.1109/CVPR.2004.1315267

[32] S. Alpert, M. Galun, R. Basri, A. Brandt, Image Segmentation by Probabilistic Bottom-Up Aggregation and Cue Integration, in IEEE Conference on Computer Vision and Pattern Recognition, 2007.

http://dx.doi.org/10.1109/cvpr.2007.383017

\section{Authors' information}

${ }^{1}$ Department of Informatics, Faculty of Information Technology, Institut Teknologi Sepuluh Nopember (ITS), Surabaya, Indonesia.

${ }^{2}$ Department of Dentomaxillofacial Radiology, Faculty of Dental Medicine, Airlangga University, Surabaya, Indonesia.

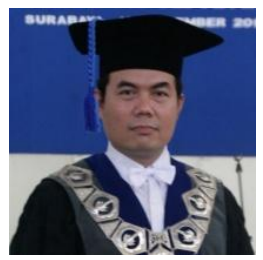

Agus Zainal Arifin has received his bachelor degree from Department of Informatics, ITS Surabaya, Indonesia in 1995. He has received his master degree from Faculty of Computer Science, University of Indonesia, Jakarta, Indonesia in 2001. He received his doctoral degree from Department of Information Engineering, Hiroshima University, Japan in 2007. Currently, he is dean of Faculty of Information Technology, ITS, Surabaya. His research interest includes computer vision, image processing, pattern recognition, and machine learning.

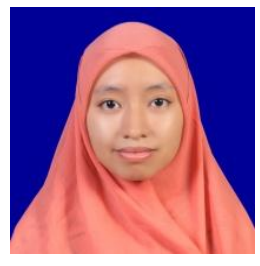

Rarasmaya Indraswari has received her bachelor degree from Department of Informatics, ITS Surabaya, Indonesia in 2015. Currently, she is a Ph.D. candidate in Computer Science, Department of Informatics, ITS Surabaya, Indonesia. Her research interest includes computer vision, image processing, pattern recognition, and machine learning. 


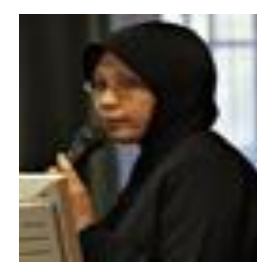

Nanik Suciati has received her B.Sc. in Computer Engineering from Teknologi Sepuluh Nopember (ITS), Indonesia in 1994. She has received her M.Sc. degree in Computer Science from University of Indonesia in 1998. She has received her Ph.D. from Hiroshima University, Japan in 2010. Since 1994 - present, she is an academic and researcher staff in Informatics Department, ITS, Indonesia. Her research interest includes computer graphics, computer vision, and image processing.

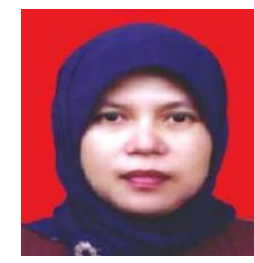

Eha Renwi Astuti has received her dental degree from Airlangga University, Surabaya, Indonesia in 1985. She received her master degree from Faculty of Public Health, Airlangga University in 1995. She received her doctoral degree from Airlangga University in 2005 Currently, she is head of Dentomaxillo Department, Facial Radiology, Faculty of Dentistry, Airlangga University, Surabaya, Indonesia.

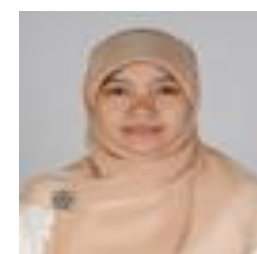

Dini Adni Navastara has received her bachelor degree from Department of Informatics, ITS Surabaya, Indonesia in 2008. She has received her M.Sc. degree from Electrical and Computer Engineering, Pusan National University, South Korea in 2014. Since 2008 - present, she is an academic and researcher staff in Informatics Department, ITS, Indonesia. Her research interest includes computer vision, image processing, pattern recognition, robotics, and machine learning. 